\title{
Interaction of Mandarin Oil with Nicorandil in Doxorubicin Induced Myocardial Infarction
}

\author{
R H Malabade* ${ }^{1}$, V P Rasal ${ }^{1}$, Ismail Pasha ${ }^{1}$ \\ ${ }^{I}$ Department of Pharmacology, KLEU's college of Pharmacy, Belgaum, Karnataka, India \\ *Rohit H Malabade \\ 'Mukta', Shastri Nagar, Bhadgaon Road, Chalisgaon, Dist- Jalgaon, State - Maharashtra, Country - India. Pin \\ code -424101
}

\begin{abstract}
Herbal cardio protective preparations are often used as an add-on therapy in cardiac disorders and such herbal preparations often contain multiple components which are responsible for Herb-Drug Interaction. Hence, the effect of mandarin oil on the pharmacokinetic and pharmacodynamic of nicorandil was studied on normal as well as on cardiotoxic male wistar rats. The influence of Mandarin oil on pharmacokinetics of nicorandil was determined by HPLC method, while pharmacodynamic interaction was studied in animals with myocardial infarction (Doxrubicin $10 \mathrm{mg} / \mathrm{kg}$, i.p, for last 2 days). Nicorandil was given i.p. at a dose of $3 \mathrm{mg} / \mathrm{kg}$ for 7 days, whereas, Mandarin oil was administered at two different doses of 200 and $400 \mathrm{mg} / \mathrm{kg}$, p.o in their respective groups for 14 days, once daily. Heart rate, ECG, Lipid profile, level of antioxidant, CK-MB, LDH, AST and ALT of heart homogenate and serum was measured and histopathological studies were carried out. Concomitant administration of mandarin oil showed a decrease $C_{\max }$ and increased $T_{\max }$ of nicornadil. All the biochemical parameters were significantly restored by nicorandil when compared with mandarin oil alone and its combination. In addition, alone and combine treatment of mandarin oil with nicorandil also improved the total antioxidant status. These finding suggests that individual treatment with nicorandil and mandarin oil provides significant cardio protection when compared to their combination in cardiotoxic rats. Hence, concomitant administration of mandarin oil along with nicorandil, should be avoided.
\end{abstract}

Keywords: Herb-Drug interaction, Nicorandil, Mandarin Oil, Cardiac enzymes, Myocardial Infarction.

\section{Introduction}

Herbal medicines have become a popular in healthcare system. All herbal medicines are mixtures of more than one active ingredient. In many cases it is unknown that, which or how many constituents are pharmacologically active. Herbal medicines co-administered along with prescribed drug tend to have chronic conditions, which increases the risk of herb-drug interaction. ${ }^{[1]}$ Interactions between herbs and drugs may increase or decrease the pharmacological and toxicological effects of either components. Synergistic therapeutic effects may complicate the dosing of long-term medications. ${ }^{[2]}$

It has been stated that $15-20 \%$ of individuals uses herbal medicines along with their prescribed medications and less than $40 \%$ of patients disclose this to their physicians. The problem is further complexed by physicians those who are not aware with the potential of herb-drug interactions. Hence it is important to promote research on the safety and efficacy of herb-drug interaction for variety of diseases including cardiovascular diseases. ${ }^{[3]}$

Nicorandil is a nicotinamide nitrate ester which is being increasingly used as the first-line and sole treatment in both stable and unstable angina and also drug approved for the treatment of ischaemic heart disease. It is believed to have dual properties of action, firstly, intrinsic mechanism of the drug i.e. selective activation of $\mathrm{K}^{+}$ATP channels at the sarcolemma and mitochondrial level. Secondly, because of $\mathrm{K}^{+}$ATP channels in ischaemic preconditioning, nicorandil has been attributed cardioprotective effects. ${ }^{[4]}$

Mandarin oil, which is obtained from peel of Citrus reticulate Balnco ${ }^{[5]}$ also called as Citrus aurantium $L$. which belong to family Rutaceae. ${ }^{[6]}$ Mandarin oil has proved effective in treating cancer ${ }^{[7]}$, hypoglycaemia ${ }^{[8]}$, also used as antiangiogenic ${ }^{[9]}$ and antifungal ${ }^{[10]}$ which states that mandarin oil has a potent antioxidant property. ${ }^{[11]}$

However, there are no scientific report to indicate the biochemical alterations, antioxidant profile, ECG parameters, hemodynamic findings and histological changes upon concomitant administration of mandarin oil along with nicorandil in DOX induced myocardial infarction. Hence, the present study was undertaken to evaluate the pharmacokinetic and pharmacodynamic interactions of nicorandil with mandarin oil in rats. 


\subsection{Chemicals and Reagents:}

\section{Methodology}

All reagents and chemicals used in this study were of analytical grade. The enzyme kits were obtained from Erba Diagnostic Manheim GmbH, Germany and Crest Biosystem, Goa. Nicorandil was obtained from local pharmacy shop while mandarin oil was obtained from local supplier (Venkateshwar supplier, Bangalore). Plasma drug concentration was measured using HPLC (Shimadzu, Serial No. L12561) and all the reagents and chemicals used were of HPLC grade.

\subsection{Experimental Animals:}

Male wistar rats (150-200g) were housed at $25 \pm 5^{\circ} \mathrm{C}$ in a well-ventilated animal house under 12:12h light $\&$ dark cycle. The rats had free access to standard rat chow (Amrut Laboratory Animal Feed, Maharashtra, India) and water ad libitum. The institutional animal ethics committee approval was taken and animals were maintained under standard conditions.

\subsection{Experimental design:}

Male Wistar rats weighing 150 - $200 \mathrm{~g}$ were used. The rats were grouped randomly into seven group of six each;

Nicorandil will be administered i.p. at a dose of $3 \mathrm{mg} / \mathrm{kg}$ for 7 days ${ }^{[13]}$ and Mandarin oil will be administered at a dose of $200 \mathrm{mg} / \mathrm{kg}$ and $400 \mathrm{mg} / \mathrm{kg}$. ${ }^{[14]}$

Group 1: received Normal saline.

Group 2: serve as disease control group received DOX (10mg/kg, i.p.) for 2 days.

Group 3: Nicorandil + DOX (10mg/kg, i.p.) for last 2 days.

Group 4: received Mandarin Oil (200mg/kg, p.o) + DOX (10mg/kg, i.p.) for 2 last days,

Group 5: received Mandarin Oil (400 mg/kg, p.o ) + DOX (10mg/kg, i.p.) for last 2 days,

Group 6: received Nicorandil + Mandarin Oil (200mg/kg, p.o) + DOX (10mg/kg, i.p.) for last 2 days,

Group 7: received Nicorandil + Mandarin Oil (400mg/kg, p.o) + DOX (10mg/kg, i.p.) for last 2 days.

Blood was withdrawn by retro-orbital puncture, serum was separated for biochemical estimation and animals were sacrificed after $48 \mathrm{hr}$. of DOX administration.

\subsection{Pharmacokinetic Study:}

Rats were treated with nicorandil $(3 \mathrm{mg} / \mathrm{kg})$ alone and in combination with mandarin oil at a dose of $200 \mathrm{mg} / \mathrm{kg}$ and $400 \mathrm{mg} / \mathrm{kg}$. Blood samples were collected from retro-orbital vein puncture using heparinised capillary tubes at 30,60 and 120 mins. Plasma was separated after centrifugation at $8000 \mathrm{rpm}$ for 15 min and stored at $-20{ }^{\circ} \mathrm{C}$.

\section{Chromatographic Condition:}

The mobile phase consisted of degassed (premixed) solution containing Methanol: Water (60:40, v/v). The mobile phase was filtered through a $0.22 \mu \mathrm{m}$-sized membrane (micropore, USA) with flow rate of $0.6 \mathrm{~mL} / \mathrm{min}$. Peak areas were determined with a spinchrome integrator. Metronidazole was adopted as an internal standard. Primary standard solutions of nicorandil and metronidazole $(100 \mu \mathrm{g} / \mathrm{mL})$ was prepared in methanol. To each plasma containing tubes, $1 \mathrm{~mL}$ of metronidazole stock solution was added. Then aliquots of $100 \mu \mathrm{L}$ of plasma samples were taken and mixed with $250 \mu \mathrm{L}$ of acetonitrile and mixtures were vortexed for 30 seconds and centrifuged at $3000 \mathrm{rpm}$ for 10 minutes. A portion of supernatants were transferred to eppendorff tubes and $20 \mu \mathrm{L}$ was injected. The eluent was detected by the UV detector at $254 \mathrm{~nm}$. Under the described conditions, the retention time and peak area of nicorandil and metronidazole, was noted down and Time vs. Plasma drug concentration graph was plotted.

Concentration of Sample was found out using below equation:

Concentration $=\frac{\text { Peak area of Sample }}{\text { Peak area of I.S }} \times \frac{\text { Concentration of Sample }}{\text { Concentration of I.S }} \times \%$ Purity of the drug

\subsection{Histopathological Evaluation:}

A portion of the heart was fixed in formalin (10\%) and subjected to histopathological studies. The section of heart was processed and embedded in paraffin wax. Sections of about 4-6 $\mu$ m were made and stained with hematoxylin and eosin at 100x and photographed. 


\subsection{Statistical Analysis}

Results are expressed as mean \pm SEM. The statistical significance was determined using one-way analysis of variance (ANOVA) followed by Dunnett's multiple comparison test.

\subsection{Pharmacodynamics parameters:}

\section{Results}

3.1.1 Cardiac marker enzyme of serum and heart tissue homogenate i.e. ALT, AST, LDH and CK-MB:

There was substantial fall $(\mathrm{p}<0.001)$ in activity of marker enzymes in heart tissue homogenate and rise $(\mathrm{p}<0.001)$ in the activities of marker enzymes in serum respectively upon DOX administration. Pre-treatment with nicorandil and mandarin oil alone significantly restored the activity to normal level in both heart homogenate and serum. But when combination was compared to nicorandil alone less significant restoration of marker enzymes was observed. (Graph no. 1 and Graph no. 2).

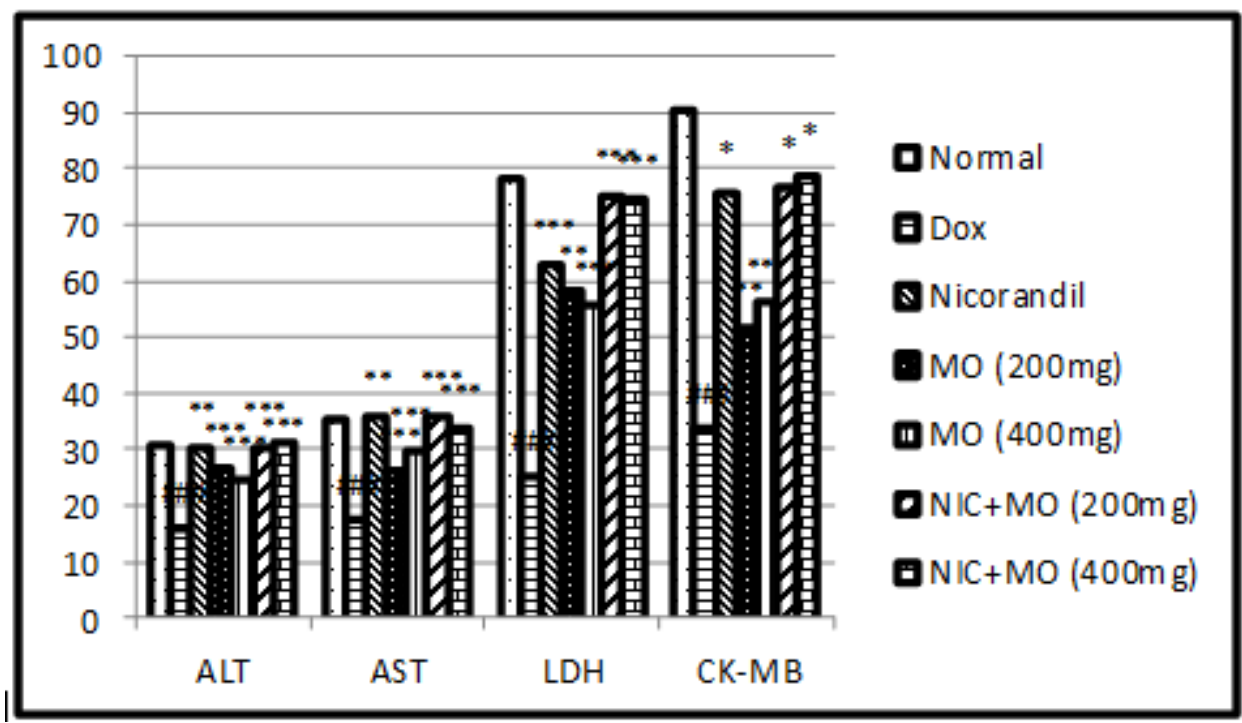

Graph no. 1: Effect of various treatments on Cardiac marker enzyme of Heart Tissue Homogenate

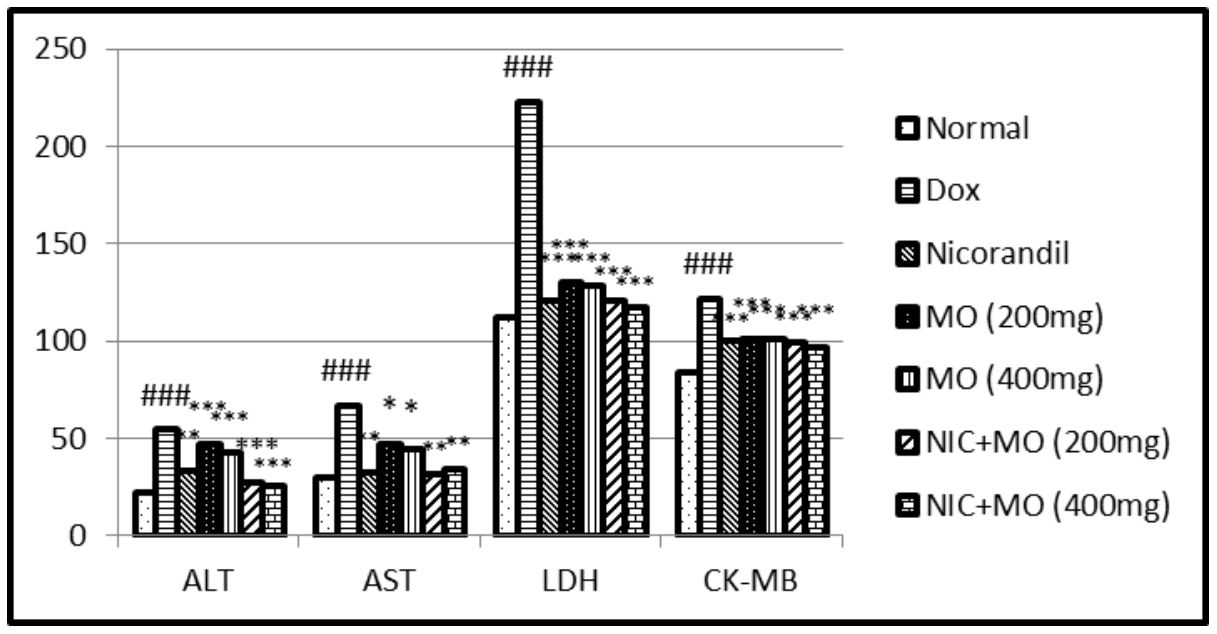

Graph no. 2: Effect of various treatments on Cardiac marker enzymes of Serum.

\subsubsection{Serum lipid Profile i.e. Total Cholesterol (T-CHO), TG, LDL, VLDL and HDL:}

Cardiotoxic rats pre-treated with nicorandil and mandarin oil alone and in combination showed significant reduction $(\mathrm{p}<0.001)$ in $\mathrm{T}-\mathrm{CHO}$ when compared with DOX induced rats. There was no significant difference in TG level observed when nicorandil and mandarin oil was given alone, but administration of nicorandil and mandarin oil in combination showed significant increase $(\mathrm{p}<0.001)$ in TG level when compared 
with DOX induced rats. Significant decrease in HDL and increase $(p<0.001)$ in LDL and VLDL was observed in DOX treated when compared to normal group and pre-treatment with nicorandil and mandarin oil alone and in combination showed significant increase in HDL and restored LDL and VLDL level when compared to DOX induced rats. (Graph no. 3)

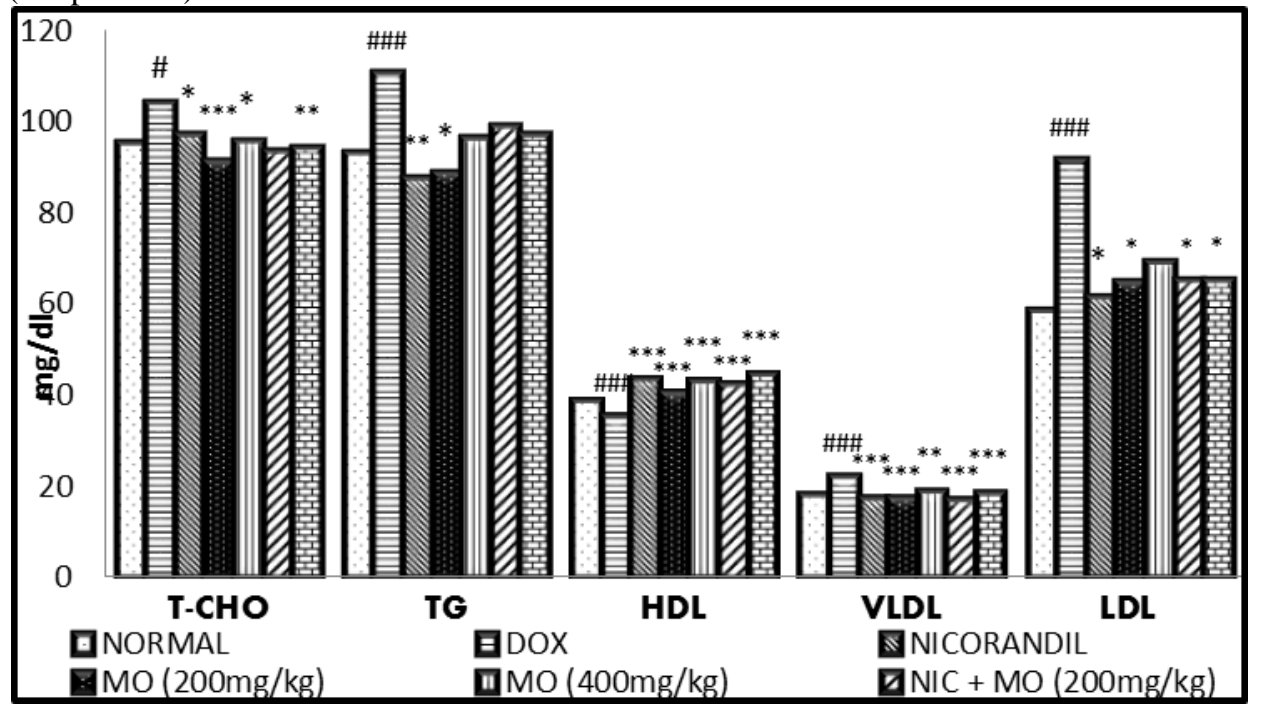

Graph No. 3: Effect of various treatments on Lipid Profile

\subsubsection{Antioxidants i.e. GSH, SOD and MDA:}

Significant decline in the activities of endogenous antioxidant enzymes was observed in the heart tissues of DOX treated rats. Pre-treatment with mandarin oil and combination with nicorandil offered significant protection $(\mathrm{p}<0.001)$ against DOX induced oxidative stress in terms of preservation of endogenous antioxidants but nicorandil alone showed less significant increase in antioxidant enzyme level when compared with DOX induced rats. The increased MDA level in DOX treated group is attribute to increase in lipid peroxidation associated tissue damage. Pre-treatment with nicorandil and mandarin oil alone and in combination appeared to have a protective effect on lipid peroxidation by decreasing $(\mathrm{p}<0.001)$ MDA level in heart tissue homogenate. (Table no. 1)

\begin{tabular}{|c|c|c|c|c|}
\hline $\begin{array}{l}\text { Sr } \\
\text { No. }\end{array}$ & GROUPS & SOD $(\mathrm{U} / \mathrm{mL})$ & $\begin{array}{c}\text { MDA }(\mu g / g \text { wet } \\
\text { tissue })\end{array}$ & $\begin{array}{c}\text { GSH }(\mu g / g \text { wet } \\
\text { tissue })\end{array}$ \\
\hline 1 & Normal & $78.52 \pm 3.80$ & $27.45 \pm 8.745$ & $971.6 \pm 9.363$ \\
\hline 2 & Doxorubicin Induced & $40.49 \pm 5.06^{\# \# \#}$ & $65.19 \pm 7.283^{\# \# \#}$ & $679.8 \pm 24.04^{\# \# \#}$ \\
\hline 3 & Nicorandil (3mg/kg) & $43.19 \pm 2.598$ & $44.87 \pm 3.581 * * *$ & $732.8 \pm 24.09$ \\
\hline 4 & $\begin{array}{l}\text { Mandarin Oil } \\
(200 \mathrm{mg} / \mathrm{kg})\end{array}$ & $64.78 \pm 4.941 * * *$ & $42.60 \pm 2.421 * * *$ & $836.6 \pm 55.07 * * *$ \\
\hline 5 & $\begin{array}{l}\text { Mandarin Oil } \\
(400 \mathrm{mg} / \mathrm{kg})\end{array}$ & $69.35 \pm 6.337 * * *$ & $38.18 \pm 7.985^{* * *}$ & $840.4 \pm 76.98 * * *$ \\
\hline 6 & $\begin{array}{c}\text { Nicorandil + } \\
\text { Mandarin Oil } \\
(3 \mathrm{mg}+200 \mathrm{mg} / \mathrm{kg})\end{array}$ & $70.09 \pm 6.312 * * *$ & $42.48 \pm 9.01 * *$ & $767.3 \pm 184.0 * *$ \\
\hline 7 & $\begin{array}{c}\text { Nicorandil + } \\
\text { Mandarin Oil } \\
(3 \mathrm{mg}+400 \mathrm{mg} / \mathrm{kg})\end{array}$ & $71.54 \pm 4.229 * * *$ & $35.63 \pm 9.18 * * *$ & $785.1 \pm 53.49$ \\
\hline
\end{tabular}

Table no. 1: Effect of various treatments on Anti-oxidant enzymes in Doxorubicin induced myocardial infarction. 
All values are expressed as mean \pm SEM for six animals in each group using one-way analysis of variance (ANOVA) followed by Dunnett's multiple comparison test. Compared with Normal control: ${ }^{\#} \mathrm{P}<0.05$, $\mathrm{P}<0.01,{ }^{\# \# \#} \mathrm{P}<0.001$. Compared with Disease control: ${ }^{*} \mathrm{P}<0.05,{ }^{* * *} \mathrm{P}<0.01,{ }^{* * * *} \mathrm{P}<0.001$.

\subsubsection{Body weight, ECG and Heart Rate:}

Present study showed that, DOX caused significant decrease in body weight. The rats pre-treated with nicorandil and mandarin oil alone and in combination showed significant increase in body weight. ECG alterations were monitored, DOX treated group showed significant prolongation of QT interval and elevation of ST segment, with significant increase in heart rate. Pre-treatment with nicorandil alone significantly reduced ECG alterations. But, concomitant pre-treatment with mandarin oil alone and in combination with nicorandil significantly decreased the alterations in cardiotoxic rats. (Table no. 2)

\begin{tabular}{|c|c|c|c|c|}
\hline $\begin{array}{c}\text { Sr } \\
\text { No. }\end{array}$ & Groups & Heart rate (bpm) & QRS complex(sec) & QT interval(sec) \\
\hline 1 & Normal & $290.3 \pm 16.11$ & $0.051 \pm 0.0012$ & $0.090 \pm 0.0015$ \\
\hline 2 & DOX Induced & $331.5 \pm 15.75^{\# \# \#}$ & $0.038 \pm 0.0028^{\# \# \#}$ & $0.113 \pm 0.0012^{\# \# \#}$ \\
\hline 3 & $\begin{array}{c}\text { Nicorandil + Dox } \\
\mathbf{( 3 m g / k g )}\end{array}$ & $272.4 \pm 13.31^{*}$ & $0.046 \pm 0.0012^{* *}$ & $0.085 \pm 0.0015^{*}$ \\
\hline 4 & $\begin{array}{c}\text { Mandarin Oil } \\
\mathbf{( 2 0 0 m g / k g ) + D o x}\end{array}$ & $250.13 \pm 14.20^{* * *}$ & $0.040 \pm 0.0028^{*}$ & $0.080 \pm 0.0015^{* *}$ \\
\hline 5 & $\begin{array}{c}\text { Mandarin Oil } \\
\mathbf{( 4 0 0 m g / k g ) + D o x}\end{array}$ & $242.9 \pm 15.41^{* * *}$ & $0.042 \pm 0.0021^{* *}$ & $0.079 \pm 0.00015^{* *}$ \\
\hline 6 & $\begin{array}{c}\mathbf{N I C}+\mathbf{M O} \\
\mathbf{( 2 0 0 m g / k g ) + D o x}\end{array}$ & $246.2 \pm 16.03^{* * *}$ & $0.043 \pm 0.0028^{* *}$ & $0.082 \pm 0.0015^{* * *}$ \\
\hline 7 & $\begin{array}{c}\mathbf{N I C + M O} \\
\mathbf{( 4 0 0 m g / k g ) + D o x}\end{array}$ & $255.5 \pm 12.34^{* *}$ & $0.042 \pm 0.0028^{* *}$ & $0.085 \pm 0.0015^{* * *}$ \\
\hline
\end{tabular}

Table no. 2: Shows effect of various treatments on ECG and Heart rate

All values are expressed as mean \pm SEM for six animals in each group using one-way analysis of variance (ANOVA) followed by Dunnett's multiple comparison test.

Compared with Normal control: ${ }^{\#} \mathrm{P}<0.05$, ${ }^{\# \#} \mathrm{P}<0.01,{ }^{\# \# \#} \mathrm{P}<0.001$. Compared with Disease control: ${ }^{*} \mathrm{P}<0.05$, ${ }^{* * *} \mathrm{P}<0.01,{ }^{* * * *} \mathrm{P}<0.001$.

\subsubsection{Pharmacokinetic Determination:}

As depicted from plasma drug concentration vs. Time graph (Graph no. 4), time required to reach peak $\left(\mathrm{T}_{\max }\right)$ plasma concentration of nicorandil alone occurred at 30 min while in combination with mandarin oil, occurred at 60 mins, whereas $\mathrm{C}_{\max }$ of nicorandil alone was $324.3 \mu \mathrm{g}$ while nicorandil in presence of mandarin oil at dose of $200 \mathrm{mg} / \mathrm{kg}$ is $196.4 \mu \mathrm{g}$ and at $400 \mathrm{mg} / \mathrm{kg}$ is $240.5 \mu \mathrm{g}$. Therefore substantially decrease in $\mathrm{C}_{\max }$ and increased $\mathrm{T}_{\max }$ indicating decreased extent of absorption of nicorandil in presence of mandarin oil. 


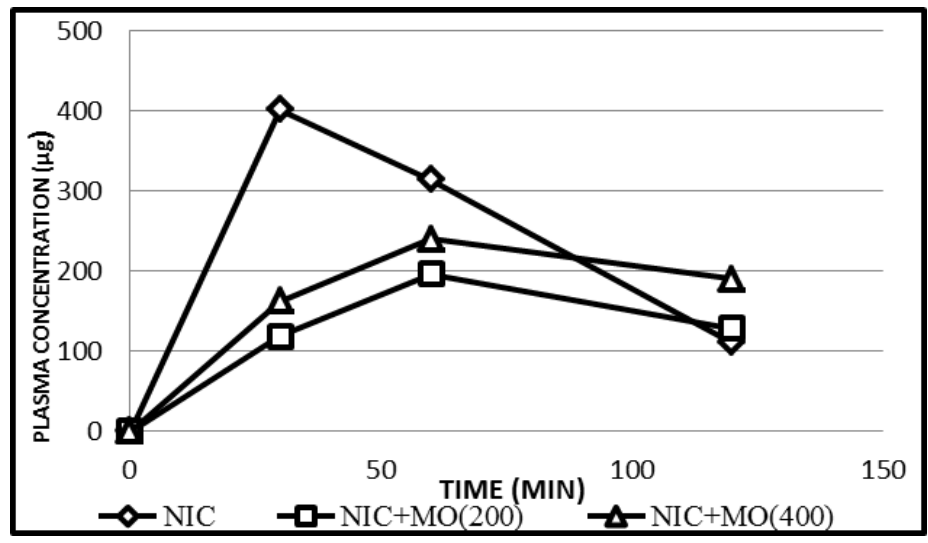

Graph no. 4: Plasma Drug Concentration vs. Time

\subsubsection{Histopathological Evaluation:}

Myocardial integrity was disturbed by administration of DOX i.p for 2 consecutive days showed degenerative changes like necrosis, vascular edema etc. Pre-treatment with nicorandil and mandarin oil alone and there combination restored normal myofibril condition. (Fig no. 1)
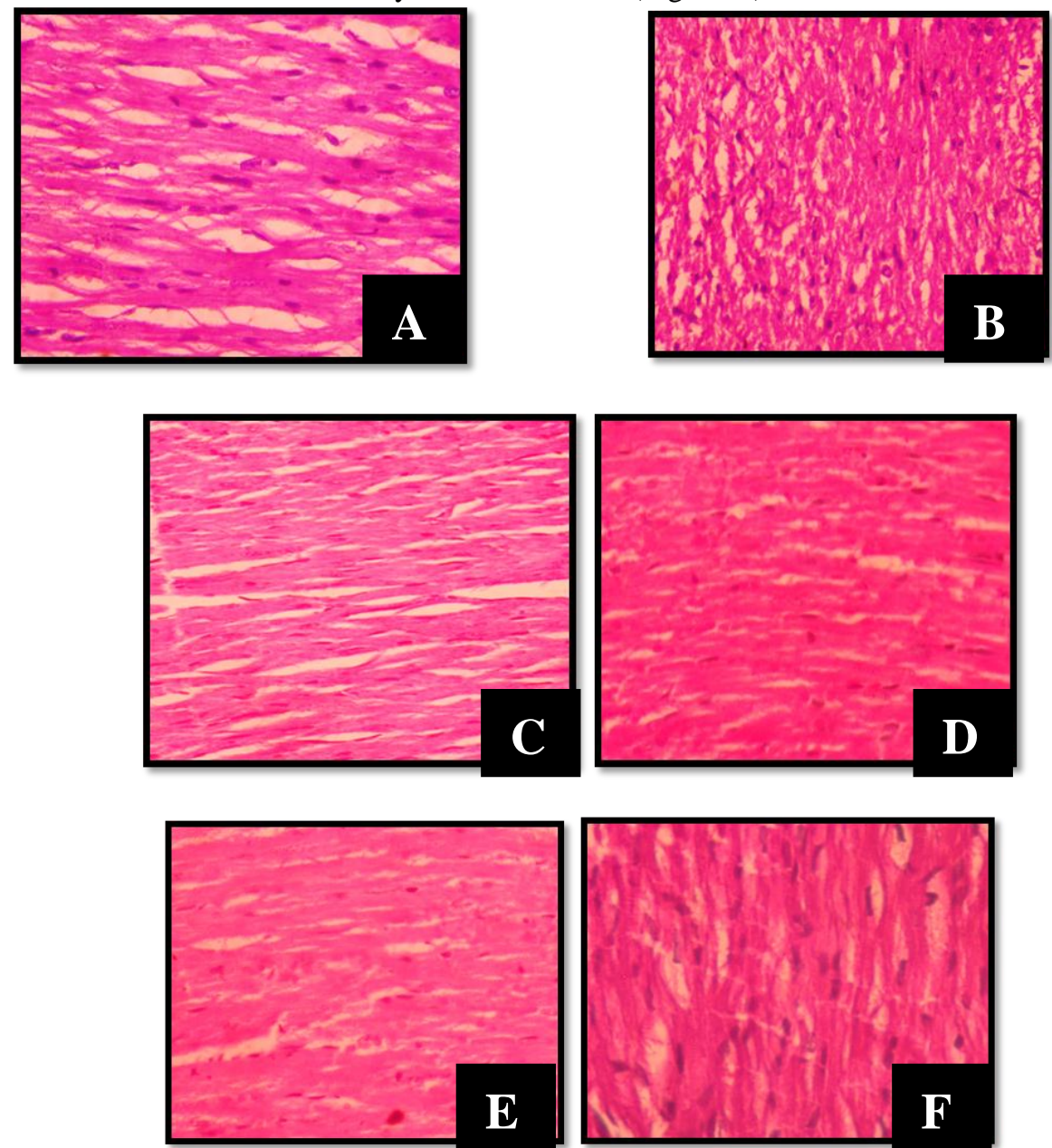


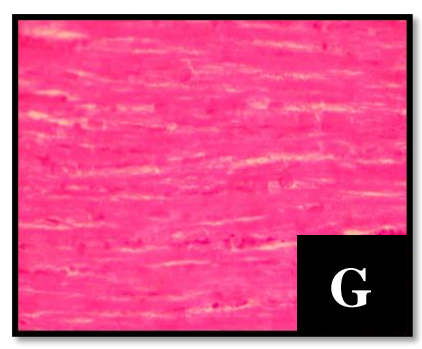

Fig No.1: Effect of various treatments on myocardial tissue

\section{Discussion}

The aim of the present study was to explore interaction of mandarin oil with nicorandil in DOX induced myocardial infarction. Results revealed the presence of pharmacodynamic and pharmacokinetic interaction when mandarin oil was administered in combination with nicorandil in cardiotoxic rats. DOX, an anthracycline antibiotic, widely used as anticancer agent. It has been believed that DOX induced cardiac injury, mainly mediated through free radical generation; iron-dependent oxidative damage to the biological macromolecules and membrane lipid peroxidation. ${ }^{[15]}$ DOX administered rats showed ECG change, free radical generation, antioxidant deficit, and increase levels of serum lipid profile, significant decrease in the activities of cardiac marker enzymes, cardiomyopathy and eventually congestive heart failure. ${ }^{[16]}$

DOX induced myocardial damage associated with decrease in endogenous antioxidants such as superoxide dismutase (SOD), GSH and MDA that are structurally and functionally impaired by free radicals resulting in damage to myocardium ${ }^{[17]}$. Elevated activity of GSH, SOD and MDA was found to be significant increase with pre-treatment of mandarin oil alone and in combination with nicorandil provides integrity of myocardium. Administration of DOX leads to damage of myocardial tissue which causes the release of marker enzymes in blood stream and depletion in myocardial tissue. This condition was pre-dominantly restored by alone treatment of nicorandil and mandarin oil. Pre-treatment with nicorandil and mandarin oil alone and in combination significantly restored lipid level and also increased HDL level.

Damage to cardiac muscle was also demonstrated and confirmed by histopathology. Nicorandil and mandarin oil alone and in combination were found to minimise the damage induced by DOX. The electrocardiographic parameters were normalized in individual therapy especially heart rate was reverted to normal conditions as well as QRS duration was reduced indicating protection from myocardial ischemia. Present study showed that individual treatment is more effective in cardioprotection and combinational treatment due to presence of pharmacodynamic interaction in mandarin oil and nicorandil. Pharmacokinetically mandarin oil was interacting with nicorandil, as observed in Plasma drug concentration vs. Time, plot, showed decrease in $\mathrm{C}_{\max }$ and increase $\mathrm{T}_{\max }$.

\section{Conclusion}

In conclusion, it can be interpreted that mandarin oil and nicorandil should be avoided in combination because of presence of pharmacodynamic and pharmacokinetic interaction, which was observed in treatment of myocardial infarction induced by DOX. Hence, further studies can be carried out to determine the influence of specific active constituent responsible for interaction of mandarin oil with nicorandil in myocardial damage. This type of study will open new areas and will be helpful in carrying out further research of interaction between herb and conventional drugs when they are taken concurrently.

\section{References}

[1]. Angelo A, Edzard E, Interactions between herbal medicines and prescribed drugs, Drugs, 61(15), 2001, $2163-75$.

[2]. Adriane F, Herb-Drug Interactions, Lancet, 8, 2000, 134-8.

[3]. Akpanabiatu M, Edet E, Ukaffia S, Effects of interaction of vitamin A and rauwolfia vomitoria root bark extract on marker enzymes of cardiac diseases, Indian Journal of Clinical Biochemistry, 24 (3), 2009, 241- 4.

[4]. Eric Eeckhouta, Nicorandil, a drug for many purposes: too good to be true, Eur Heart J, 24, 2003, $1282-4$.

[5]. Ambasto SP, The Useful plants of India. Publication \& Information Directorate, 1986:129.

[6]. Vaidyasala A, Indian Medicinal Plant. Orient Longman. Vol 2, 1994, 108-9.

[7]. Mervat MA, Gendy EA, In vitro, evaluation of medicinal activity of egyptian honey from different floral sources as anticancer and antimycotic infective agents, JMBT, 2(5), 2010, 118-23.

[8]. Gavimath CC, Kangralkar VA, Jadhav NA, Burli SC, Effect of essential oil of Citrus reticulata on blood glucose and lipid profile in alloxan induced diabetic rats, Int J Pharmaceut Appl, 1(1), 2010, 1-5.

[9]. Chrisnanto E, Adelina R, Dewi DPP, Sahid MNA, Setyaningtias D, Jenie RI, Meiyanto E, Antiangiogenic effect of ethanol extract of Citrus reticulata peel in the chorio allantoic membrane (CAM) induced by bFGF, Proceeding Molecular Targeted Therapy Symposium, 2008; 57-66

[10]. Chutia M, Bhuyan PD, Pathak MG, Sarma TG, Boruah P, Antifungal activity and chemical composition of Citrus reticulata blanco essential oil against phytopathogens from north east India, Lebenson Wiss Technol, 42, 2009, 777-80. 
[11]. Ghasemi K, Ghasemi Y, Ebrahimzadeh MA, Antioxidant activity, phenol and flavonoid contents of 13 citrus species peels and tissues, Pak J Pharm Sci, 22(3), 2009, 277-81.

[12]. Jahangir A, Terzic A, KATP channel therapeutics at the bedside, J Mol Cell Cardiol 39, 2005, 99-112.

[13]. Kangralkar VA, Gavimath CC, Vijapur V, Gowri B, Hooli VR, Mathapati P, Protective effect of essential oil of Citrus reticulata on isoniazid induced hepatotoxicity in wistar rats, Int J Pharmaceut Appl 1(2), 2010,59-61.

[14]. Chlebowski RT, Adriamycin (Doxorubicin) cardiotoxicity: a review, West J Med 131, 1979, 364-8.

[15]. Iliskovic N, Singal P K, Lipid lowering: an important factor in preventing adriamycin-induced heart failure, Am J Pathol, 150(2), 1997, 727-34.

[16]. Basheeruddin A, Inamdar N, Pharmacodynamic and Pharmacokinetic interactions of propranolol with garlic (Allium sativum) in Rats, Evidence-Based Complementary and Alternative Medicine, 2011: 1-11. 